\title{
Evolutionary history of the genus Tarentola (Gekkota: Phyllodactylidae) from the Mediterranean Basin, estimated using multilocus sequence data
}

\author{
Catarina Rato ${ }^{1,2,3^{*}}$, Salvador Carranza ${ }^{3}$ and David J Harris ${ }^{1,2}$
}

\begin{abstract}
Background: The pronounced morphological conservatism within Tarentola geckos contrasted with a high genetic variation in North Africa, has led to the hypothesis that this group could represent a cryptic species complex, a challenging system to study especially when trying to define distinct evolutionary entities and address biogeographic hypotheses. In the present work we have re-examined the phylogenetic and phylogeographic relationships between and within all Mediterranean species of Tarentola, placing the genealogies obtained into a temporal framework. In order to do this, we have investigated the sequence variation of two mitochondrial (12S rRNA and 165 rRNA), and four nuclear markers (ACM4, PDC, MC1R, and RAG2) for 384 individuals of all known Mediterranean Tarentola species, so that their evolutionary history could be assessed.

Results: Of all three generated genealogies (combined mtDNA, combined nDNA, and mtDNA+nDNA) we prefer the phylogenetic relationships obtained when all genetic markers are combined. A total of 133 individuals, and 2,901 bp of sequence length, were used in this analysis. The phylogeny obtained for Tarentola presents deep branches, with T. annularis, T. ephippiata and T. chazaliae occupying a basal position and splitting from the remaining species around 15.38 Mya. Tarentola boehmei is sister to all other Mediterranean species, from which it split around 11.38 Mya. There are also two other major groups: 1) the T. mauritanica complex present in North Africa and Europe; and 2) the clade formed by the T. fascicularis/deserti complex, T. neglecta and T. mindiae, occurring only in North Africa. The cladogenesis between these two groups occurred around 8.69 Mya, coincident with the late Miocene. Contrary to what was initially proposed, $T$. neglecta and $T$. mindiae are sister taxa to both $T$. fascicularis and T. deserti.

Conclusions: At least in the Iberian Peninsula and Northwest Africa, the lineages obtained have some geographic coherency, whilst the evolutionary history of the forms from Northeast Africa remains unclear, with a paraphyletic T. fascicularis with respect to $T$. deserti. The separation between the T. mauritanica complex and the clade formed by the T. fascicularis/deserti complex, T. neglecta and T. mindiae is coincident with the uplift of the Atlas Mountain chain, and the establishment of two distinct bioclimatic regions on each side of the barrier.
\end{abstract}

\section{Background}

The Mediterranean Basin has been the stage of various paleogeographical events that have helped shape the genetic diversity and phylogeographic patterns of numerous taxa. In particular, the connection between the

\footnotetext{
* Correspondence: catarina.rato@mail.icav.up.pt

'CIBIO, Centro de Investigação em Biodiversidade e Recursos Genéticos,

Campus Agrário de Vairão, 4485-661 Vairão, Portugal Full list of author information is available at the end of the article
}

Mediterranean Sea and the Atlantic Ocean closed about 5.96 million years ago (Mya) causing the Mediterranean Sea to desiccate during the Messinian Salinity Crisis, an event that ended 5.33 Mya, with the formation of the Strait of Gibraltar [1-3]. The opening of the Strait led to the separation of terrestrial faunal elements in the region into allopatric units [3], as in the cases of the spinyfooted lizards Acanthodactylus erythrurus [4], or the West Mediterranean newts of the genus Pleurodeles [5].
C Biomed Central 
Regarding the study performed on the spiny-footed lizards, the Iberian Acanthodactylus e. erythrurus apparently became separated from its North African relatives around 5 Mya [4], and it was also the refilling of the Strait of Gibraltar that may have led to the separation between Pleurodeles waltl and the North African newts Pleurodeles nebulosus and Pleurodeles poireti [5]. Another extremely influential event occurred during the Pleistocene when sea level and climate oscillations led to repeated isolation and connection of taxa [6,7], and consequent population differentiation [e.g. [8,9]]. At a more local scale, the Atlas Mountains originated during the mid- to late Miocene as a consequence of the impact between the Eurasian and African plates [10], and seem to have been an important barrier for allopatric speciation [e.g. [11,12]]. All these events have made the Mediterranean Basin a centre for genetic differentiation that, in some cases, has led to vicariant speciation, partially explaining the observed high levels of biodiversity [13,14].

Tarentola geckos are members of the Family Phyllodactylidae [15], and the genus is currently comprised of 21 different species [16-20]. Tarentola is distributed across the Mediterranean Basin and on many Macaronesian islands, including Madeira, the Selvages, the Canary and Cape Verde islands $[21,22]$. On the other side of the Atlantic Ocean, three species are accepted: T. americana, from Cuba and the Bahamas; the recently described T. crombiei [18] endemic to Cuba; and the probably extinct $T$. albertschwartzi [16], known from a single specimen allegedly from Jamaica. Since all the Macaronesian islands are volcanic, and therefore have never been connected to the mainland [23], the most likely hypothesis is that these geckos reached these islands by transmarine dispersal $[21,22]$. Regarding the Neotropical members of Tarentola, these also seem to be the result of a postGondwanan dispersal from the Old World [21,24,25].

The Mediterranean Basin harbours nine different species of Tarentola, namely T. mauritanica, T. chazaliae, T. deserti, T. boehmei, T. annularis, T. neglecta, T. mindiae, T. ephippiata, and T. fascicularis, a former subspecies of $T$. mauritanica recently elevated to the species level [17]. Although several molecular studies have been published for some of these species, these have always been focused on one [26-30] or a few taxa [17,19,21,24,25]. Moreover, all species of Mediterranean Tarentola have never been assembled into a phylogenetic context using a multilocus approach. The majority of the studies have been focused on the phylogeographic patterns of the Moorish gecko T. mauritanica. This species is characterized by an extremely high mitochondrial genetic variation in North Africa, which led to the hypothesis that this taxon could be, in fact, a species complex $[29,30]$. In contrast, the European populations of the Moorish gecko have very low mitochondrial diversity, initially thought to be the result of a recent introduction [21,28-30], probably human-mediated. However, a recent study [26] has demonstrated that this low mtDNA variability that characterizes the European populations of T. mauritanica could be the result of genetic hitch-hiking, and not solely due to a recent colonization event.

Additionally, several studies have demonstrated the paraphyly of $T$. mauritanica with respect to $T$. angustimentalis from the Canary Islands [21,26-30]. A recent study [17] concluded that populations of $T$. fascicularis from Libya and central Tunisia constitute a monophyletic lineage and, together with $T$. mindiae and T. neglecta, represent the sister group of $T$. deserti. On the other hand, previous mtDNA studies indicated that $T$. fascicularis is paraphyletic with respect to $T$. deserti [26-30], although sampling was limited in all cases.

Therefore, in order to estimate an evolutionary hypothesis able to explain the previously mentioned patterns we have increased the taxonomic and geographic sampling in this study. Further, we used two mitochondrial, and four nuclear markers in order to better evaluate the inter- and intraspecific relationships within the genus Tarentola from the Mediterranean Basin.

\section{Results}

\section{mtDNA genealogy}

With the program ALTER [31] the 384 concatenated mitochondrial sequences were reduced to 125 haplotypes used in the analyses. A total of $818 \mathrm{bp}$ (132 variable sites), corresponding to $301 \mathrm{bp}$ of $12 \mathrm{~S}$ rRNA and $517 \mathrm{bp}$ of $16 \mathrm{~S}$ rRNA, were used. According to jModelTest the model of nucleotide substitution that best fits the $12 \mathrm{~S}$ rRNA dataset is the SYM+I+G, and for the $16 \mathrm{~S}$ rRNA it is the $\mathrm{HKY}+\mathrm{G}$. According to the obtained mitochondrial DNA genealogy results (Figure 1), we were able to recover six major groups within the Mediterranean species of Tarentola, geographically represented in Figure 2; one group corresponding to T. chazaliae; another one clustering $T$. annularis and T. ephippiata; T. boehmei appears as two separated lineages; another clade comprised of T. neglecta and T. mindiae, sister taxa to both $T$. deserti and T. fascicularis; and another group clustering T. mauritanica and T. angustimentalis with this latter rendering the former paraphyletic, as previously reported [21,26-30]. Within these major divisions T. chazaliae is basal to all Mediterranean Tarentola and T. annularis and T. ephippiata are sister taxa. Within the T. boehmei group considerable diversity was obtained, with one lineage from southwestern Morocco appearing as an independent evolutionary entity, sister to all remaining Tarentola, although this relationship was not statistically supported. In fact, the southwestern Moroccan clade of $T$. boehmei is the only one presenting strong geographic coherency, with the remaining lineages of this species 


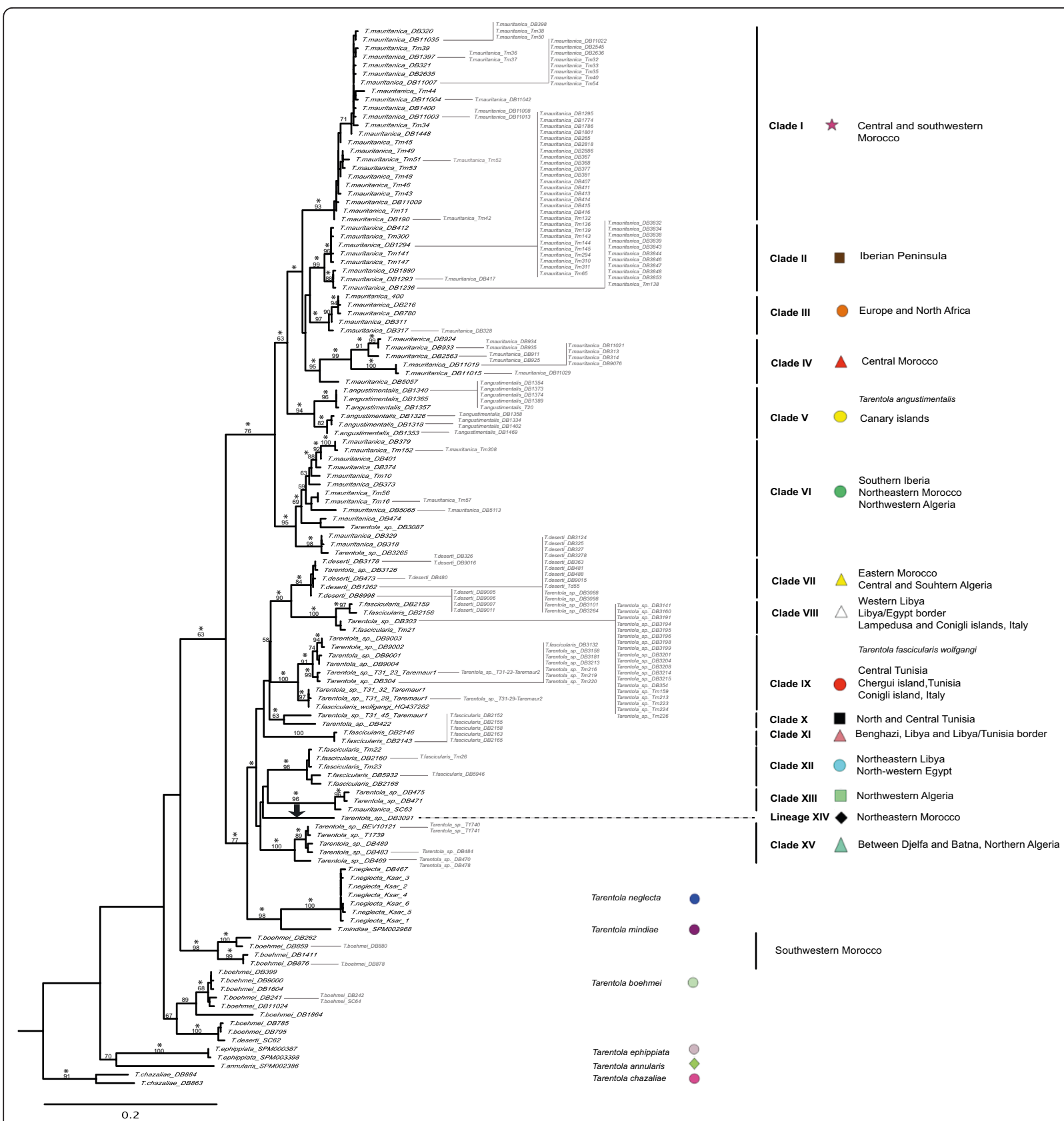

Figure 1 RAxML mtDNA phylogenetic tree for the combined 12S rRNA and 16S rRNA. Maximum likelihood bootstrap scores are represented over key nodes, and the star symbol corresponds to Bayesian posterior probabilities higher than 95\%. The black arrow corresponds to incongruences between both phylogenetic methods, with DB3091 appearing as sister taxa to Clade XII, with Bayesian Inference, also weakly supported (53\%).

clustering specimens from different geographic localities. A further feature of the T. boehmei lineage is the existence within this clade of a specimen assigned as $T$. deserti (SC62) by Carranza et al. [19]. In fact, SC62 was collected from the same locality as $\mathrm{Td} 55$, a specimen morphologically identified as $T$. deserti, and that falls within the $T$. deserti clade [29], and far away from the distribution range of $T$. boehmei. Unfortunately, there is no available information for the nuclear markers of this specimen, in order to determine if this is a case of hybridization, mitochondrial introgression between $T$. boehmei and $T$. deserti, a misidentification (implying a substantial range extension) or some other form of error.

The group comprised by T. mauritanica and T. angustimentalis can be subdivided into six different evolutionary clades (I to VI); Clade I is present in Central and 


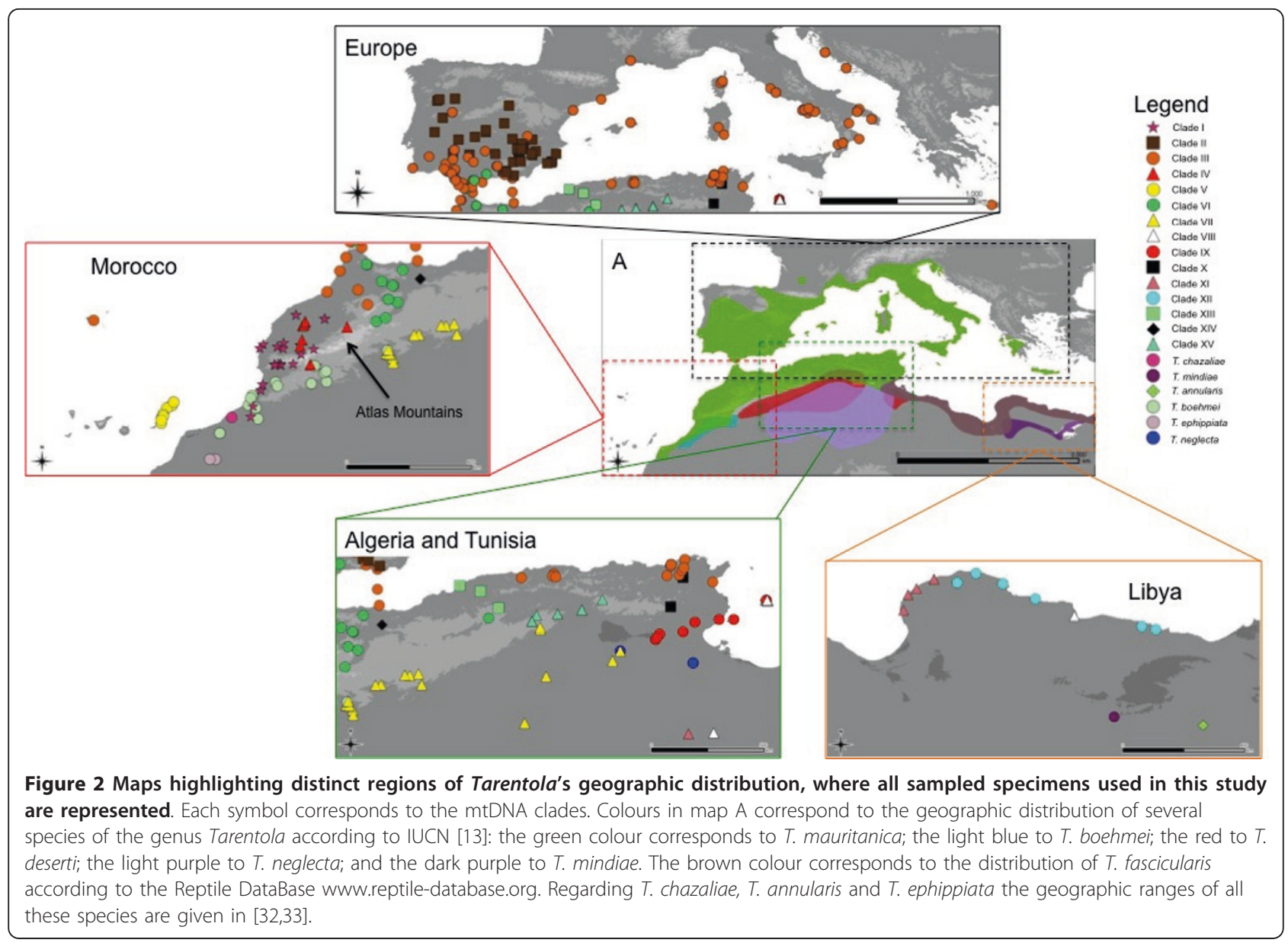

southwestern Morocco; Clade II corresponds to the exclusive Iberian lineage first identified by Perera and Harris [28]; Clade III is present throughout Europe and North Africa; a new clade assigned as Clade IV occupies Central Morocco; the T. angustimentalis (Clade V) lineage within the T. mauritanica complex; and finally Clade VI distributed across southern Iberia, northeastern Morocco and northwestern Algeria. Hereafter, this major group is referred to as the "T. mauritanica complex".

When analysing the last major lineage, there is evidence that $T$. neglecta and T. mindiae are sister taxa to both $T$. fascicularis and T. deserti, rather than the previous estimate of relationships [17], in which T. neglecta, together with $T$. mindiae and $T$. fascicularis, were sister taxa to $T$. deserti. A paraphyletic T. fascicularis with respect to T. deserti, as already obtained in previous studies, [26-30] was also recovered. This major group that hereafter is referred to as the "T. fascicularis/deserti complex" was subdivided into nine different lineages (from VII to XV), characterized by little geographical and clear phylogenetic structure. Specimens from Clade VII occur from eastern Morocco to Central and southern Algeria, fitting the known distribution of $T$. deserti [32,33]. In fact, the specimens from this study collected in the Algerian Sahara (DB481 and DB488) are located in the north and south border of the type locality of this species (Ouargla, Algerian Sahara) [34]. The sister lineage of Clade VII is Clade VIII present in western Libya, Lampedusa and Conigli islets (Italy), and on the border between Libya and Egypt, morphologically assigned to T. fascicularis (P. Geniez, pers. comm.). Clade IX ranges from Central Tunisia to Chergui island (Tunisia) and Conigli islet in which the recently described subspecies $T$. fascicularis wolfgangi [17] from central Tunisia clusters with the remaining specimens. Clade X ranges from North to Central Tunisia, and Clade $\mathrm{XI}$ is present in the Benghazi region (Libya), and on the Libya/Tunisian border. The type locality of $T$. fascicularis is Ain Teyanah, $20 \mathrm{~km}$ South of Benghazi [35], and specimens from Clade XI are included in this area. Clade XII is present in northeastern Libya and northwestern Egypt, while specimens from Clade XIII occur in northwestern Algeria. Lineage XIV is represented by a unique individual (DB3091) from northeastern Morocco, and Clade XV occurs in northern Algeria between Djelfa and Batna.

This same topology was obtained with both phylogenetic methods, except regarding the specimen DB3091 
that appears as sister taxon to Clade XII with the Bayesian Inference (BI).

\section{nDNA genealogy}

Concerning the phylogenetic inference with solely the four nuclear markers (Figure 3), we used a total of 133 individuals, with a combined length of 2,084 bp; $368 \mathrm{bp}$ for the ACM4, $602 \mathrm{bp}$ for the MC1R, $363 \mathrm{bp}$ for the PDC, and $751 \mathrm{bp}$ for the RAG2. The nucleotide models obtained for each gene were the GTR+G for the ACM4, the $\mathrm{GTR}+\mathrm{I}+\mathrm{G}$ for both MC1R and RAG2, and the K80 $+G$ for the PDC. The topologies obtained from both phylogenetic methods (ML and BI) were exactly the same, except regarding the specimen coded as DB311, which appears as sister taxon of Clades II and III in the BI analyses, and the clade clustering $T$. annularis and T. ephippiata, which is estimated to be the sister taxa of all other ingroup lineages using BI.

From the analysis of the nuclear dataset, four of the six major lineages identified in the mitochondrial phylogeny are recovered. However, the phylogenetic relationships within each of these lineages are quite different, relative to the ones obtained with the mtDNA dataset, and several of them are poorly supported with multiple polytomies. According to the ML analyses, T. ephippiata and T. annularis are sister taxa to $T$. chazaliae, although poorly supported. Also, the two mtDNA independent lineages of T. boehmei are recovered as monophyletic and contrary to the mtDNA phylogeny is considerably supported $(70 \%$ bootstrap). The Iberian clade (Clade II) within Clade III renders the latter paraphyletic and, therefore, they could be considered a single lineage based on the nDNA data. Clade VI appears as the sister taxa of both Clades II and III, instead of Clade IV. Relationships between Clades I, IV and $\mathrm{V}$ are unresolved. The group comprised by the T. fascicularis/deserti complex, T. neglecta and T. mindiae contains a single individual from Clade I (Tm11) that is more related to these taxa, rather than to the specimens from the T. mauritanica complex. This major group is characterized by several very poorly supported lineages. Clade VII is recovered, although an individual from Clade VIII (DB3204) makes this lineage paraphyletic. The T. mindiae and $T$. neglecta clade is again recovered as a lineage, with an individual from Clade X (DB422) as sister taxon to this lineage. Finally, all North Algerian, and northeastern Moroccan mtDNA lineages (clades XIII, XIV, and XV) were recovered as a distinct lineage by the $\mathrm{nDNA}$.

\section{Combined analysis (mtDNA+nDNA) and age estimates}

In order to perform this analysis (Figure 4 and Additional file 1, Figure S1), we used a total of 133 individuals, and 2,901 bp (817 bp of two mtDNA genes, and $2,084 \mathrm{bp}$ of four nDNA genes). All lineages identified with the mtDNA dataset were recovered, although some of the relationships between them were altered. According to this genealogy, T. annularis and T. ephippiata appear as sister taxa, in turn related to $T$. chazaliae. These three species split from the remaining Mediterranean Tarentola approximately 15.38 (11.53-19.93) Mya. The $T$. boehmei lineage appears as monophyletic and originated around 11.38 (8.53-14.28) Mya. Within the T. mauritanica complex, Clade $\mathrm{I}$ is sister taxon to Clades II, III and IV, and all six lineages from this major group originated between 5.88 (4.37-7.54) and 2.47 Mya (1.41-3.53), corresponding to the late Miocene and late Pliocene, respectively. As obtained from the mtDNA dataset alone, Clade VI is the sister taxa to all remaining lineages from this species complex. Tarentola neglecta and $T$. mindiae are sister taxa to the $T$. fascicularis/ deserti complex, and the split between these groups occurred around 7.04 (6.29-8.86) Mya. The phylogenetic relationships within the $T$. fascicularis/deserti complex remain unresolved, and with little geographic correspondence, although all North Algerian clades (Clades XIII, and $\mathrm{XV}$ ) were clustered together, and represent a distinct lineage. The cladogenesis between the $T$. mauritanica complex and the clade formed by the T. fascicularis/ deserti complex plus T. mindiae and T. neglecta took place around 8.69 (6.62-10.94) Mya, corresponding to the mid-late Miocene.

\section{Discussion}

In light of the ages obtained for the node estimation, it is clear that the genealogy of the North African Tarentola dates back to the Miocene, with some speciation events predating most of the known paleogeographic occurrences that took place in the western Mediterranean. This is true regarding the early split of the ancestor of the lineage formed by $T$. annularis, $T$. chazaliae and T. ephippiata, and for the origin of T. boehmei. Interestingly, the split between the T. mauritanica complex and its sister clade formed by the ancestor of $T$. neglecta, $T$. mindiae and the $T$. fascicularis/deserti complex, was around 8.69 Mya (mid-late Miocene), which is coincident with the period of uplift of the Atlas Mountain chain [10]. The establishment of this geographic barrier may have separated the ancestor of the T. mauritanica complex to the western part of the Atlas, subject to a semiarid and sub-humid bioclimatic region, while the other major lineage could have been isolated eastwards, and confined to a more arid and Saharan typical habitat. Later, around 7.04 Mya the common ancestor of $T$. neglecta and $T$. mindiae separated from the $T$. fascicularis/deserti complex. The maintenance of Tarentola species adapted to a Saharan climate still occurs today, with $T$. deserti, $T$. neglecta and $T$. mindiae all occurring in drier regions than T. mauritanica $[20,32,33]$. The vicariant effect of the 


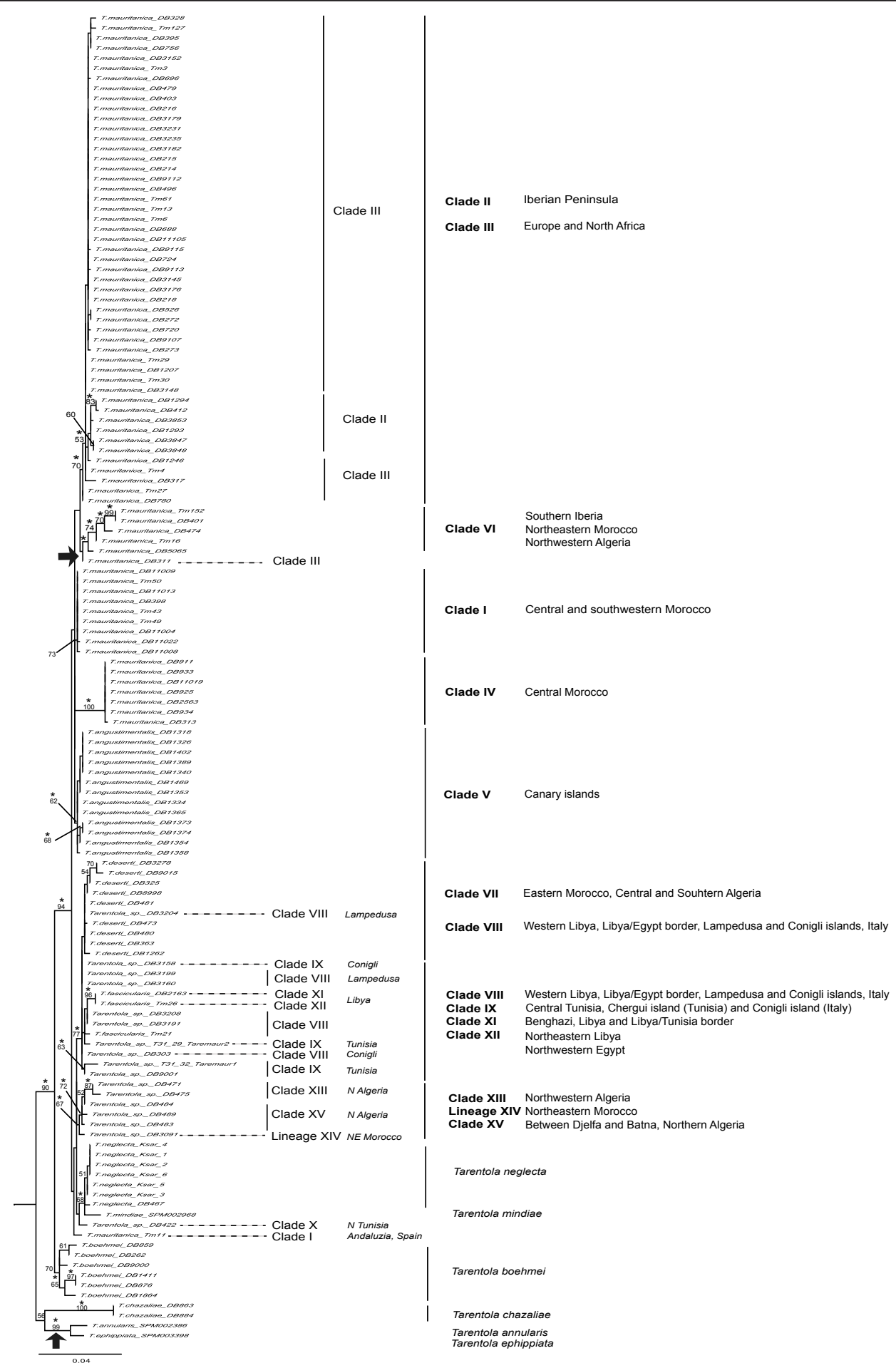

Figure 3 RAxML phylogenetic tree for all concatenated nuclear markers, namely ACM4, MC1R, PDC, and RAG2. Maximum likelihood bootstrap scores are represented over key nodes, and the star symbol corresponds to Bayesian posterior probabilities higher than $95 \%$. The black arrows correspond to incongruences between both phylogenetic methods, with DB311 appearing as sister taxon to Clades II and III (with $67 \%$ of posterior probability), and T. annularis and T. ephippiata (without T. chazaliae) with a basal position in the phylogeny, according to Bayesian Inference. According to the BI method, T. chazaliae is separated from the remaining Tarentola with a $64 \%$ of posterior probability. 


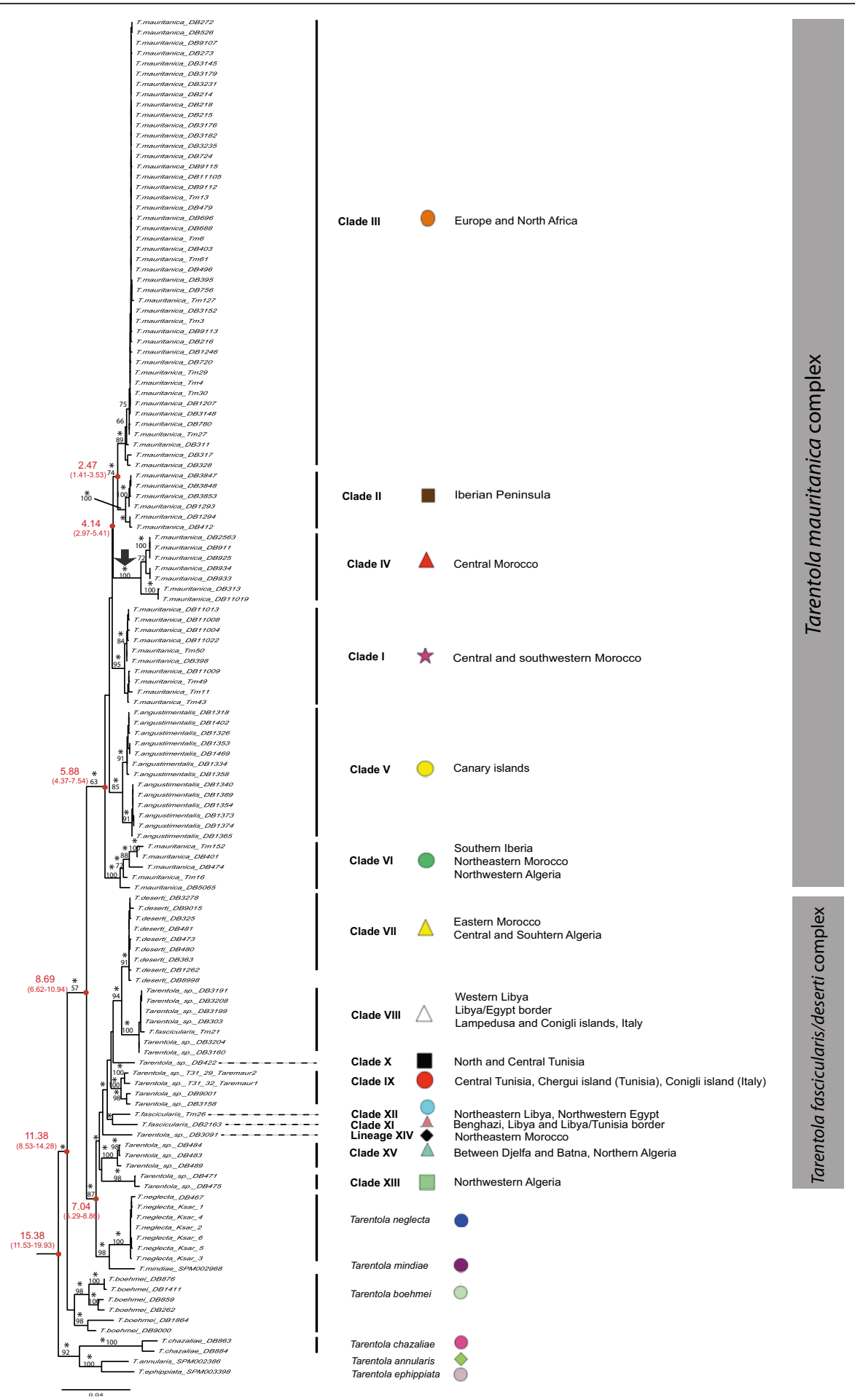

Figure 4 RAxML phylogenetic tree for all markers used in this study (mtDNA+nDNA). Maximum likelihood bootstrap scores are represented over key nodes, and the star symbol corresponds to Bayesian posterior probabilities higher than 95\%. Below the nodes, and in red are represented the estimates (mean and 95\% credibility interval) obtained of the time to the most recent common ancestor (TMRCA). The black arrow corresponds to incongruences between both phylogenetic methods, with Clade IV appearing as a politomy and sister taxa to Clades I and $\checkmark$, according to the Bayesian Inference. 
Moroccan Atlas Mountains has also been detected in other reptile species, such as Agama impalearis [11] and Mauremys leprosa [12].

The connection between the Mediterranean Sea and the Atlantic Ocean was interrupted during the late Miocene in a period that started 5.96 Mya and ceased 5.33 Mya, causing the Messinian Salinity Crisis [1-3]. This event led to the establishment of a land bridge between Africa and Europe and an opportunity for faunistic interchange, as reported for the spiny-footed lizard Acanthodactylus erythrurus [4], the snake Natrix maura [36], the skink Chalcides bedriagae [37] and the newts of the genus Pleurodeles [5]. Regarding the T. mauritanica complex, cladogenesis between Clade VI and the remaining lineages (5.88 Mya) matches very closely the closing of the Strait of Gibraltar, and is contemporary to the Messinian Salinity Crisis. According to our node age estimates, the separation between Clade IV (Central Morocco) and the ancestor of both clades II (Iberia) and III (Europe) occurred during the early Pliocene, around 4.14 Mya, after the opening of the Strait of Gibraltar, although the confidence interval could cover this period (up to 5.41 Mya). Therefore, considering the confidence interval of this node estimate, the colonization of the Iberian Peninsula by Tarentola from North Africa could have been during the Messinian Salinity Crisis, or after the opening of the Strait of Gibraltar during the early Pliocene. The crossing of the Strait of Gibraltar after it reopened has already been documented for the Podarcis wall lizards [38-40] and Psammodromus algirus [41].

Our results recover a well-supported mitochondrial phylogenetic tree for Tarentola from North Africa, adding considerably to what was previously known concerning this genus $[17,19,26-30]$. However, mitochondrial gene trees can be misleading particularly due to historical hybridization and introgression [e.g. [42]], and data from multiple unlinked loci should be used to infer phylogenetic history. Our nuclear phylogeny, while limited in resolving power, with several polytomies for the most recent nodes, did recover four of the six major lineages identified with the mtDNA alone.

Although, much debate has surrounded the validity of T. angustimentalis as a full species [21,26-30], our results from the nuclear DNA do support this taxon as an independent lineage within the T. mauritanica complex. Therefore, the recognition of $T$. angustimentalis implies that the T. mauritanica group is, in fact, a complex of several distinct forms that need to be recognized as full species. However, we suggest that elevation of independent lineages to specific status should only be done after an assessment of morphological variation, and detailed sampling to fully determine their distribution. Various recent studies have highlighted how taxonomic changes can be premature when cryptic diversity occurs, even if prior geographic sampling is extensive [e.g. [38]]. The primary objective of this work is not to make taxonomic revisions, but to assess the phylogenetic relationships within and between Mediterranean Tarentola.

According to the combined analysis, a monophyletic $T$. deserti was recovered, but this group renders $T$. fascicularis as a paraphyletic assemblage. It seems likely that T. fascicularis is actually a complex of lineages, some of which may deserve specific status. In order to unravel their evolutionary relationships, additional sampling and a morphological evaluation are required, especially across the western part of T. fascicularis' distribution range, and further identification of possible hybrids and contact zones. Regarding the recovered North Algerian lineages (XV and XIII), at least for clade XV there is already morphological evidence that this is, in fact, a distinct species (Julien Viglione, pers. comm.). If so, our phylogenetic data gives support to this as a monophyletic lineage.

\section{Conclusions}

Mediterranean Tarentola are a complex of species complexes whose differentiation was initiated in the middle Miocene. The advent of multilocus approaches has, in general, caused researchers to move away from a strict reliance on gene trees, and it is now recognized that the signal from one gene tree is less important than the sum of signals across gene trees and loci [43]. Therefore, we support the phylogeny obtained by the combined data, which supports some already accepted relationships, but also indicates various novel ones:

(1) T. annularis and T. ephippiata are sister taxa, and $T$. chazaliae appears as a sister taxon to this pair of species. These three species differentiated from the remaining Tarentola during the Miocene, around 15.38 Mya.

(2) T. boehmei presents considerable genetic variation, and is sister taxon to all remaining North African lineages, comprised by the T. mauritanica complex, $T$. fascicularis/deserti complex, T. neglecta, and T. mindiae. The cladogenesis of T. boehmei occurred around 11.38 Mya.

(3) Regarding the T. mauritanica complex, Clade VI is sister taxon to all remaining lineages [26-30], whose differentiation took place around 5.88 Mya.

(4) A new lineage within the T. mauritanica complex was identified (Clade IV) from Central Morocco, sister taxon of Clades II and III.

(5) T. angustimentalis is recovered as a monophyletic lineage within the $T$. mauritanica complex.

(6) T. neglecta and T. mindiae form a sister clade to a complex of $T$. deserti and $T$. fascicularis, as opposed to the relationships recovered by Joger and Bshaenia [17]. The ancestor of both $T$. neglecta and T. mindiae got split from their sister taxa about 7.04 Mya. 
(7) T. fascicularis is paraphyletic with respect to T. deserti.

(8) Two North Algerian clades (XV and XIII) are recovered, that are weakly supported as the sister taxa to the remaining specimens of the $T$. fascicularis/desert $i$ complex.

(9) The separation between the T. mauritanica complex and the clade comprised by the ancestor of T. neglecta, T. mindiae and the T. fascicularis/deserti complex, occurred around 8.69 Mya (mid-late Miocene), which is coincident with the period of uplift of the Atlas Mountain chain.

This study has considerably refined the major groups that exist, and at least in the Iberian Peninsula and Northwestern Africa has defined their biogeographic patterns. The situation in Northeastern Africa remains more obscure. Detailed morphological and modelling approaches, combined with further sampling will be needed to ensure that taxonomic changes can be made in order to reflect the complex evolutionary history of the group.

\section{Methods}

\section{DNA extraction, amplification and sequencing}

Tissue from tail tip muscle was collected from each individual and preserved in $96 \%$ ethanol. Genomic DNA was extracted using the DNeasy Extraction Kit from Qiagen following the manufacturer's protocol. A total of 384 individuals were used, belonging to all known species of Tarentola in the Mediterranean Basin. Some of the individuals included in this study were already used in previous works $[17,26-30,44,45]$. Geographic location of each specimen is represented in Figure 2, and detailed information about locality and amplified genes in Table S1 from Additional file 2.

For all individuals the Polymerase Chain Reaction (PCR) amplification and sequencing of two mtDNA gene fragments, the 12SrRNA and 16SrRNA was performed using the primers $12 \mathrm{Sa} / 12 \mathrm{Sb}$ and $16 \mathrm{Sar} / 16 \mathrm{Sbr}$ from Kocher et al. [46] and Palumbi [47], respectively. PCR conditions were the same as those described in Harris et al. [48]. Four nuclear protein-coding gene fragments were also sequenced: the acetylcholinergic receptor M4 (ACM4), the melanocortin 1 receptor (MC1R), the phosducin (PDC), and the recombination activating gene 2 (RAG2). For amplification and sequencing of ACM4 the primers tg-F and tg-R published by Gamble et al. [24] were used. Regarding the MC1R fragment the primers MC1R_F and MC1R_R from Pinho et al. [49] were used, and primers PHOF2 and PHOR1 [50] for the amplification and sequencing of PDC. Amplification of ACM4, MC1R and PDC fragments were carried out in $25 \mu \mathrm{l}$ volumes, containing $5.0 \mu \mathrm{l}$ of $10 \mathrm{x}$ reaction Buffer, $2.0 \mathrm{mM}$ of $\mathrm{MgCl} 2,0.5 \mathrm{mM}$ each dNTP, $0.2 \mu \mathrm{M}$ each primer, $1 \mathrm{U}$ of Taq DNA polymerase (Invitrogen), and approximately $100 \mathrm{ng}$ of template DNA. Finally, amplification and sequencing of the RAG2 gene fragment was performed using two sets of primers; 31FN.Venk/ Lung.460R (amplification) and Lung.35F/Lung.320R (amplification and sequencing) published by Hoegg et al. [51]. PCR conditions were the same as described in Chiari et al. [52]. All amplified fragments were sequenced in a ABI3730XL automated sequencer.

The obtained sequences were imported into the software Geneious Pro v5.4.0 [53] where alignment was performed with MAFFT v6.814b [54] using the default parameters (auto algorithm; scoring matrix = $200 \mathrm{PAM} /$ $\mathrm{k}=2$; gap open penalty $=1.53$; and offset value $=$ $0.123)$. All sequences generated in this study were submitted to GenBank with accession numbers ranging from JQ300539 to JQ301443. Detailed information on the individuals and sequences are described in the Table S1 from Additional file 2.

\section{Gene genealogies, and age estimates}

Using the software ALTER [31] the mtDNA dataset was reduced to unique haplotypes, considering gaps as differences. Regarding the nuclear loci, heterozygous positions were coded with the corresponding ambiguity letter.

In order to determine the best fitting nucleotide model for each gene (mtDNA and nDNA), we used the software jModelTest v0.1.1 [55], under the Akaike Information Criterion [following [56]]. Maximum Likelihood (ML), and Bayesian Inference (BI) phylogenetic analyses were performed for both the concatenated mitochondrial and nuclear DNA datasets, and for all the loci (mtDNA + nDNA). ML analyses were conducted with the software RAxML v7.2.8 alpha [57], partitioning the dataset per locus. For all analyses 20 thorough ML searches were performed in order to obtain the best ML tree with support values, and thereafter 1000 bootstrap inferences. A majority rule consensus tree was generated using the software Phyutility [58]. BI was implemented with the program Mr.Bayes v3.1.2 [59] under a partitioned model (dataset divided into genes), and considering the model of nucleotide substitution estimated with jModelTest. The Bayesian posterior probabilities were estimated using a Metropolis-coupled Markov chain Monte Carlo sampling approach, and both runs started with random trees running for $10 \times 10^{6}$ generations, saving one tree every 100 generations producing 100,000 trees. Both convergence and appropriate sampling were confirmed by examining the standard deviation of the split frequencies between the two simultaneous runs and the Potential Scale Reduction Factor (PSRF) diagnostic. The first 25,000 trees of each run were included in the burn-in period and discarded. Next, a majority-rule consensus 
tree was generated from the remaining trees. In both phylogenetic analyses Ptyodactylus hasselquistii was used as outgroup.

The T. boettgeri group from the Canary and Selvages Islands has been used in previous analyses to provide a calibration point for estimates of the time of the cladogenetic events for the phylogeny of this genus $[19,21]$, but the lack of available nuclear DNA sequences precludes its use in this study As an alternative, the substitution rate of the same mitochondrial region calculated for Tarentola was used for this purpose. Mean substitution rates and the standard error of the mean values for exactly the same $12 \mathrm{~S}$ region as in the present study was extracted from a fullycalibrated phylogeny of Tarentola from the Canary islands $[19,21]$. This value was used as an informative prior in our divergence dating analysis. Specifically, we set a normal distribution prior for the ucld.mean parameter of the $12 \mathrm{~S}$ partitions based on the result of the meanRate posterior (mean and standard error) of the calibration analyses of Tarentola $(0.00891 \pm 0.0000376$ for the $12 S)$.

We used BEAST v.1.6.1 [60] to estimate dates of the cladogenetic events from the concatenated dataset. The dataset comprised sequences from all six genes (nuclear genes unphased) using a phylogeny pruned arbitrarily to include one representative from each of the major lineages uncovered with the concatenated analysis (29 specimens in total). This method excludes closely related terminal taxa because the Yule tree prior (see below) does not include a model of coalescence, which can complicate rate estimation for closely related sequences [61]. Analyses were run four times for $5 \times 10^{7}$ generations with a sampling frequency of 10,000. Models and prior specifications applied were as follows (otherwise by default): GTR $+\mathrm{I}+\mathrm{G}(12 \mathrm{~S}$, 16S), TN93 (ACM4), HKY+G (MC1R), HKY (PDC), TN93+G (RAG2); Relaxed Uncorrelated Lognormal Clock (estimate); Yule process of speciation; random starting tree; alpha Uniform $(0,10)$; yule.birthRate $(0,1000)$; ucld. mean of $12 \mathrm{~S}$ Normal (initial value: 0.00827, mean: 0.00827, Stdev: 0.00162). Convergence for all model parameters was assessed by examining trace plots and histograms in Tracer v1.4 [62] after obtaining an effective sample size $(E S S)>200$. The initial $10 \%$ of samples were discarded as burn-in. Runs were combined using LogCombiner, and maximum credibility trees with divergence time means and $95 \%$ highest probability densities (HPDs) were produced using Tree Annotator (both part of the BEAST package). Trees were visualized using FigTree v1.3.1 (available at http://tree.bio.ed.ac.uk/software/figtree).

\section{Additional material}

Additional file 1: Figure S1. Node age estimates among each mtDNA clade for the combined dataset, using the software BEAST v.1.6.1 and considering a relaxed uncorrelated lognormal clock and the Yule process of speciation as tree prior. Estimation of the used substitution rate is explained in the text.

Additional file 2: Table S1. Table with information regarding the locality, and sequenced genes for all specimens used in this study. The specimens coded with a star have the same mitochondrial haplotype as individual T. mauritanica_400.

\section{Acknowledgements and funding}

CR is supported by the PhD grant SFRH/BD/36387/2007 from the Fundação para a Ciência e Tecnologia (FCT). Research was supported by the FCT projects PTDC/BIA-BDE/74349/2009 and by the Ministério de Educación y Ciencia, Spain: CGL2009-11663/BOS (to SC). SC and CR are part of the Grup de Recerca Emergent of the Generalitat de Catalunya: 2009SGR1462. The authors are grateful to many colleagues who helped collect samples in the field, in particular to S. Larbes (Tizi-Ouzou University, Algeria) and P. Lymberakis (Natural History Museum of Crete, Greece) who donated samples from their collection. Also, the authors acknowledge P. Geniez and J. Viglione (both from ECO-MED-Ecologie et Médiation, France) for helping in the identification of several North African specimens. Samples were collected in Spain under permits SGYB-AFR-CMM, CMUmjglase, and AUT/ CAP/ASO/57/09, and in Morocco with the permit 14HCEFLCD/DLCDPN/ DPRN/CFF. The authors would also like to acknowledge the anonymous reviewers of this work, whose suggestions were of extreme importance.

\section{Author details}

${ }^{1} \mathrm{CIBIO}$, Centro de Investigação em Biodiversidade e Recursos Genéticos, Campus Agrário de Vairão, 4485-661 Vairão, Portugal. ${ }^{2}$ Departamento de Biologia, Faculdade de Ciências da Universidade do Porto, 4099-002 Porto, Portugal. ${ }^{3}$ Institute of Evolutionary Biology (CSIC-UPF)-Passeig Marítim de la Barceloneta, 37-49, E-08003 Barcelona, Spain.

\section{Authors' contributions}

CR carried out the entire molecular laboratory work, analysed the data and drafted the manuscript. All authors participated in the conception and design of the study, collection of samples, writing and approval of the final manuscript.

Received: 19 October 2011 Accepted: 30 January 2012

Published: 30 January 2012

\section{References}

1. Krijgsman W, Hilgen FJ, Raffi I, Sierro FJ, Wilson DS: Chronology, causes and progression of the Messinian salinity crisis. Nature 1999, 400:652-655.

2. Duggen S, Hoernle K, van den Bogaard P, Rupke L, Morgan JP: Deep roots of the Messinian salinity crisis. Nature 2003, 422:602-606.

3. Hsü KJ, Montadert L, Bernoulli D, Cita MB, Erickson A, Garrison RE, Kidd RB, Mèlierés F, Müller $C$, Wright R: History of the Mediterranean salinity crisis. Nature 1977, 267:399-403.

4. Harris DJ, Batista V, Carretero MA: Assessment of genetic diversity within Acanthodactylus erythrurus (Reptilia: Lacertidae) in Morocco and the Iberian Peninsula using mitochondrial DNA sequence data. AmphibiaReptilia 2004, 25:227-232.

5. Carranza S, Arnold EN: History of West Mediterranean newts, Pleurodeles (Amphibia: Salamandridae), inferred from old and recent DNA sequences. Syst Biodivers 2004, 1(3):327-337.

6. Hewitt G: Some genetic consequences of the ice ages and their role in divergence and speciation. Biol J Linn Soc 1996, 58:247-266.

7. Hewitt G: Postglacial recolonization of the European biota. Biol J Linn Soc 1999, 68:87-612.

8. Rato C, Zuffi MAL, Corti C, Fornasiero S, Gentilli A, Razzetti E, Scali S, Carretero MA, Harris DJ: Phylogeography of the European Whip Snake, Hierophis viridiflavus (Colubridae), using mtDNA and nuclear DNA sequences. Amphibia-Reptilia 2009, 30:283-289.

9. Santos X, Roca J, Pleguezuelos JM, Donaire D, Carranza S: Biogeography and evolution of the Smooth snake Coronella austriaca (Serpentes: Colubridae) in the Iberian Peninsula: evidence for Messinian refuges and Pleistocenic range expansions. Amphibia-Reptilia 2008, 29:35-47. 
10. Gomez F, Barazangi M, Beauchamp W: Role of the Atlas Mountains northwest Africa within African-Eurasian plate-boundary zone. Geology 2000, 28:769-864.

11. Brown RP, Suaréz NM, Pestano J: The Atlas Mountains as a biogeographical divide in North-West Africa: evidence from mtDNA evolution in the Agamid lizard: Agama impalearis. Mol Phylogenet Evol 2002, 24:324-332.

12. Fritz U, Fritzsch G, Lehr E, Ducotterd J-M, Müller A: The Atlas Mountains, not the Strait of Gibraltar, as a biogeographic barrier for Mauremys leprosa (Reptilia: Testudines). Salamandra 2005, 41(3):97-106.

13. Cox N, Chanson J, Stuart S, Compilers: The Status and Distribution of Reptiles and Amphibians of the Mediterranean Basin. Gland, Switzerland and Cambridge, UK: IUCN; 2006.

14. Myers N, Mittermeier RA, Mittermeier CG, da Fonseca GAB, Kent J: Biodiversity hotspots for conservation priorities. Nature 2000, 403:853-858.

15. Gamble T, Bauer AM, Greenbaum E, Jackman TR: Out of the blue: a novel, trans-Atlantic clade of geckos (Gekkota, Squamata). Zool Scr 2008, 37(4):355-366.

16. Sprackland RG, Swinney GN: A new species of giant gecko of the genus Tarentola (Reptilia: Squamata: Gekkonidae) from Jamaica. J Zool London 1998, 245:73-78

17. Joger U, Bshaenia I: A new Tarentola subspecies (Reptilia: Gekkonidae) endemic to Tunisia. Bonn Zoological Bulletin 2010, 57(2):267-274.

18. Díaz LM, Hedges SB: A new gecko of the genus Tarentola (Sauria: Gekkonidae), from Eastern Cuba. Zootaxa 2008, 1743:43-52.

19. Carranza S, Arnold EN, Mateo JA, Geniez M: Relationships and evolution of the North African geckos, Geckonia and Tarentola (Reptilia: Gekkonidae), based on mitochondrial and nuclear DNA sequences. Mol Phylogenet Evol 2002, 23:244-256.

20. Baha El Din SM: A new species of Tarentola (Squamata: Gekkonidae) from the Western Desert of Egypt. Afr J Herpetol 1997, 46(1):30-35.

21. Carranza S, Arnold EN, Mateo JA, López-Jurado LF: Long-distance colonization and radiation in gekkonid lizards, Tarentola (Reptilia: Gekkonidae), revealed by mitochondrial DNA sequences. Proc $R$ Soc Lond B 2000, 267:637-649.

22. Vasconcelos R, Carranza S, Harris DJ: Insight into an island radiation: the Tarentola geckos of the Cape Verde archipelago. J Biogeogr 2010, 37(6):1047-1060

23. Schmincke H-U: Geology of the Canary Islands. In Biogeography and Ecology in the Canary Islands. Edited by: Kunkel G. The Hague: The Netherlands: W. Junk; 1976:

24. Gamble T, Bauer AM, Greenbaum E, Jackman TR: Evidence for Gondwanan vicariance in an ancient clade of gecko lizards. J Biogeogr 2008, 35:88-104.

25. Gamble T, Bauer AM, Colli GR, Greenbaum E, Jackman TR, Vitt LJ, Simons AM: Coming to America: multiple origins of New World geckos. J Evolution Biol 2011, 24:231-244.

26. Rato C, Carranza S, Perera A, Carretero MA, Harris DJ: Conflicting patterns of nucleotide diversity between mtDNA and nDNA in the Moorish gecko, Tarentola mauritanica. Mol Phylogenet Evol 2010, 56:962-971.

27. Harris DJ, Carretero MA, Corti C, Lo Cascio P: Genetic affinities of Tarentola mauritanica (Reptilia: Gekkonidae) from Lampedusa and Conigli islet (SW Italy). North-West J Zool 2009, 5(1):197-205.

28. Perera A, Harris DJ: Genetic diversity in the gecko Tarentola mauritanica within the Iberian Peninsula. Amphibia-Reptilia 2008, 29:583-588.

29. Harris DJ, Batista V, Lymberakis P, Carretero MA: Complex estimates of evolutionary relationships in Tarentola mauritanica (Reptilia: Gekkonidae) derived from mitochondrial DNA sequence. Mol Phylogenet Evol 2004, 30:855-859.

30. Harris DJ, Batista V, Carretero MA, Ferrand N: Genetic variation in Tarentola mauritanica (Reptilia: Gekkonidae) across the Strait of Gibraltar derived from mitochondrial and nuclear DNA sequences. Amphibia-Reptilia 2004 25:451-459.

31. Glez-Peña D, Gómez-Blanco D, Reboiro-Jato M, Fdez-Riverola F, Posada D: ALTER: program-oriented format conversion of DNA and protein alignments. Nucl Acids Res 2010, 38(Suppl 2):W14-W18

32. Schleich HH, Kästle W, Kabisch K: Amphibians and reptiles from North Africa. Königstein, Germany: Koeltz Scientific Publications; 1996.

33. Bons J, Geniez P: Amphibians and Reptiles of Morocco. Barcelona, Spain: Asociación Herpetológica Española; 1996, 319.
34. Boulenger GA: Catalogue of the reptiles and batrachians of Barbary (Morocco, Algeria, Tunisia), based chiefly upon the notes and collections made in 1880-1884 by M. Fernand Lataste. Tr Zool Soc 1891, 13:93-164.

35. Joger U: Taxonomisch Revision der Gattung Tarentola (Reptilia: Gekkonidae). Zool Beitr 1984, 35:129-174.

36. Guicking D, Lawson R, Joger U, Wink M: Evolution and phylogeny of the genus Natrix (Serpentes: Colubridae). Biol J Linn Soc 2006, 87:127-143.

37. Carraza S, Arnold EN, Geniez P, Roca J, Mateo JA: Radiation, multiple dispersal and parallelism in the skinks, Chalcides and Sphenops (Squamata: Scincidae), with comments on Scincus and Scincopus and the age of the Sahara Desert. Mol Phylogenet Evol 2008, 46:1071-1094.

38. Kaliontzopoulou A, Pinho C, Harris DJ, Carretero MA: When cryptic diversity blurs the picture: a cautionary tale from Iberian and North African Podarcis wall lizards. Biol J Linn Soc 2011, 103:779-800.

39. Pinho C, Ferrand N, Harris DJ: Reexamination of the Iberian and North African Podarcis (Squamata: Lacertidae) phylogeny based on increased mitochondrial DNA sequencing. Mol Phylogenet Evol 2006, 38:266-273.

40. Harris DJ, Carranza S, Arnold EN, Pinho C, Ferrand N: Complex biogeographic distribution of genetic variation within Podarcis Wall lizards across the Strait of Gibraltar. J Biogeogr 2002, 29(9):1257-1262.

41. Carranza S, Harris DJ, Arnold EN, Batista V, Gonzales de la Vega JP: Phylogeography of the lacertid lizard, Psammodromus algirus, in Iberia and across the Strait of Gibraltar. J Biogeogr 2006, 33:1279-1288.

42. Leaché AD, McGuire JA: Phylogenetic relationsips of horned lizards (Phrynosoma) based on nuclear and mitochondrial data: Evidence for a misleading mitochondrial gene tree. Mol Phylogenet Evol 2006, 39:628-644.

43. Dolman G, Moritz C: A multilocus perspective on refugial isolation and divergence in rainforest skinks (Carlia). Evol Int J Org Evol 2006, 60:573-582.

44. Jesus J: First record of Tarentola mauritanica (Linnaeus, 1758) on Porto Santo Island. Herpetozoa 2008, 20(3/4):175-177.

45. Ceacero F, García-Muñoz E, Pedrajas L, Perera A, Carretero MA: Tarentola and other gekkonid records from Djebel Ouarkziz (SW Morocco). Acta Herpetologica 2010, 5(1):13-17.

46. Kocher TD, Thomas WK, Meyer A, Edwards SV, Pääbo S, Villablanca FX, Wilson AC: Dynamics of mitochondrial DNA evolution in animals: amplification and sequencing with conserved primers. Proc Natl Acad SCi USA 1989, 86:6196-6200.

47. Palumbi SR: Nucleic acids II: the polymerase chain reaction. In Molecular Systematics. Edited by: Hillis D, Moritz C, Mable BK. Sunderland, MA: Sinauer; 1996:205-247.

48. Harris DJ, Arnold EN, Thomas RH: Relationships of the lacertid lizards (Reptilia: Lacertidae) estimated from mitochondrial DNA sequences and morphology. Proc R Soc Lond B 1998, 265:1939-1948.

49. Pinho C, Rocha S, Carvalho BM, Lopes S, Mourão S, Vallinoto M, Brunes TO, Haddad CFB, Gonçalves H, Sequeira F, et al: New primers for the amplification and sequencing of nuclear loci in a taxonomically wide set of reptiles and amphibians. Conserv Genet Resour 2010, 2(1):181-185.

50. Bauer AM, de Silva A, Greebaum E, Jackman T: A new species of day gecko from high elevation in Sri Lanka, with a preliminary phylogeny of Sri Lankan Cnemaspis(Reptilia, Squamata, Gekkonidae). Mitt Mus Natkd Berl, Zool 2007, 83:22-32.

51. Hoegg S, Vences M, Brinkmann H, Meyer A: Phylogeny and comparative substitution rates of frogs inferred from sequences of three nuclear genes. Mol Biol Evol 2004, 21:1188-1200.

52. Chiari $Y$, Vences $M$, Vieites DR, Rabemananjara F, Bora $P$, Ravoahangimalala OR, Meyer A: New evidence for parallel evolution of colour patterns in Malagasy poison frogs (Mantella). Mol Ecol 2004, 13:3763-3774.

53. Drummond AJ, Ashton B, Buxton S, Cheung M, Cooper A, Duran C, Field M, Heled J, Kearse M, Markowitz S, et al: Geneious v5.4, Available from [http://www.geneious.com/]. 2010.

54. Katoh K, Misawa K, Kumar K, Miyata T: MAFFT: a novel method for rapid multiple sequence alignment based on fast Fourier transform. Nucl Acids Res 2002, 30(14):3059-3066.

55. Posada D: jModelTest: Phylogenetic Model Averaging. Mol Biol Evol 2008, 25:1253-1256.

56. Posada D, Buckley TR: Model selection and model averaging in phylogenetics: advantages of Akaike Information Criterion and Bayesian approaches over likelihood ratio tests. Syst Biol 2004, 53:793-808. 
57. Stamatakis A: RAxML-VI-HPC: Maximum Likelihood-based phylogenetic analyses with thousands of taxa and mixed models. Bioinformatics 2006 22(21):2688-2690

58. Smith SA, Dunn CW: Phyutility: a phyloinformatics tool for trees, alignments and molecular data. Bioinformatics 2008, 24:715-716.

59. Ronquist F, Huelsenbeck JP: MRBAYES 3: Bayesian phylogenetic inference under mixed models. Bioinformatics 2003, 19:1572-1574.

60. Drummond AJ, Rambaut A: BEAST: Bayesian evolutionary analysis by sampling trees. BMC Evol Biol 2007, 7(214).

61. Ho SYW, Phillips MJ, Cooper A, Drummond AJ: Time dependency of molecular rate estimates and systematic overestimation of recent divergence times. Mol Biol Evol 2005, 22(7):1561-1568.

62. Rambaut A, Drummond AJ: Tracer v1.4, Available from [http://beast.bio. ed.ac.uk/Tracer]. 2007.

doi:10.1186/1471-2148-12-14

Cite this article as: Rato et al:: Evolutionary history of the genus

Tarentola (Gekkota: Phyllodactylidae) from the Mediterranean Basin, estimated using multilocus sequence data. BMC Evolutionary Biology 2012 12:14.

\section{Submit your next manuscript to BioMed Central} and take full advantage of:

- Convenient online submission

- Thorough peer review

- No space constraints or color figure charges

- Immediate publication on acceptance

- Inclusion in PubMed, CAS, Scopus and Google Scholar

- Research which is freely available for redistribution

Submit your manuscript at www.biomedcentral.com/submit 